\title{
The research of image segmentation algorithms based on Vilenkin- Crestensen functions
}

\author{
Boris V. Kostrov ${ }^{1,}$ a , Sergey I. Babaev ${ }^{1}$, Anastasia G. Svirina ${ }^{1}$ \\ ${ }^{1}$ Ryazan state radioengineering university, Electronic computing machines department, 390005 Ryazan, Russia
}

\begin{abstract}
The problem of Vilenkin-Crestenson functions (VCF) application to process aerospace images is considered. Theoretical grounds of VCF basis and its construction in the field of m-based numeric systems with operation of inversion by Walsh are discribed. Examples of basic functions system building on the basis of VCF with different bases and their application to image segmentation are shown. Avdvantages of using image segmentation algorithm based on VCF basis are discribed. Band pass spectrum restriction possibilities to highlight borders and edge the regions of similar density are given. Different forms of VCF spectrum restriction and its combinations with thier impact to image segmentation results are shown. Technical vision system application of sugested algorithm are shown. VCF basis abbility to highlight objects to be used in correlation-extreme navigation systems are shown. The experimental results of using VCF in comparasing with traditional Fourie basis are given.
\end{abstract}

\section{Introduction}

This paper is connected with general trends to develop aviation technology of the fifth generation as well as with the improvement of computer processing information systems from technical vision systems (TVS) of flight apparatus (FA) that allows to take a fresh look at the mathematical apparatus, which has been so far only of fundamental theoretical interest. Vilenkin-Crestenson functions known since the second half of the $20^{\text {th }}$ century are considered to be such mathematical apparatus.

The increased role of vision systems used together with navigation and FA flight parameters and a priori information presented in the form of digital district maps (DDM) together with increased performance of onboard computing means distinguishes the current stage of aviation science and technology development, which is characterized by growing implementation of various management system types [1-3] in complex objects the activity of which is related to the analysis of a great number of parameters of constantly changing environment. This characterization fully corresponds to modern aircraft design.

The task of building autonomous navigation systems and correlation-extreme navigation systems (CESN) and navigation by means of geophysical fields are of the same type.

\section{Theoretical grounds}

Efficient use of correlation methods with a combination of images is connected with a number of difficulties relating to the large volume of computational operations

\footnotetext{
a Corresponding author: kostrov.b.v@evm.rsreu.ru
}

$[4,5]$ and low productivity of onboard systems. In the basic approach to the construction of CESN the phase of preconditioning images processing is used in order to remove redundancy information and to select the elements being the most suitable for correlation algorithms.

This stage is commonly understood as the task of image segmentation [6], by allocating on it uniform brightness regions. The traditional scheme for determining the boundaries based on the methods of spatial differentiation involves the following steps:

- to contrast the image;

-to form a binary image.

A significant disadvantage of such multistage schemes of image processing is the large number of computational operations consequently leading to low performance.

The use of discrete exponential bases to construct extremal correlation algorithms allows to reduce information redundancy of correlated images using the filtering of respective spectral components. Using the mechanism of correlation and filtering in VilenkinCrestenson function space based on using the theorems of correlation and convolution it is necessary to introduce the elements of image matrix in the form of $\mathrm{N}$ real-valued periodic sequences of data. In this case, properties of correlation and convolution are the same as the properties of cyclic correlation and convolution for discrete Fourier transform [7] (DFT). 


\subsection{Vilenkin-Crestnson functions}

Generally, VCF function is a complex function which can be defined as:

$$
\operatorname{VKF}(p, x)=w^{\sum_{i=1}^{n} p_{i} x_{i}},
$$

where $w=e^{2 \pi j / m}, p_{i}$ and $x_{i}$ are the digits of integers $p$ and $x$, represented in $m$-based numeric system.

In the simplest case, when $m=2$ and $w=\mathrm{e}^{\pi j}=-1$ $\mathrm{VCF}$ is represented by Walsh function system. In another case, if $n=1$, the domain of functions definition is represented as $N=m^{n}=m$ so that VCF transforms to discrete exponential functions $-\operatorname{def}(p, x)=e^{\frac{2 \pi j}{N} p x}$.

VCF is the function of two equal variables $p$ and $x$, so any conclusion concerning the one variable can be equally attributed to the other. Consequently, the VCF matrix is symmetric.

VCF is a periodic function with the period of $N$ :

$$
V K F(p, x \pm N)=V K F(p, x)
$$

Vilenkin-Crestenson system of functions is multiplicative:

$$
V K F(p, x) V K F(q, x)=V K F(p+q, x)
$$

Average value of any VCF with $p \neq 0$ is 0 :

$$
\sum_{x=0}^{N-1} V K F(p, x)=\left(e^{\frac{2 \pi j}{N} p N}-1\right) /\left(e^{\frac{2 \pi j}{N} p}-1\right)
$$

VCF system is orthogonal:

$$
\sum_{x=0}^{N-1} V K F(p, x) V K F(q, x)=\left\{\begin{array}{l}
0, p \neq q \\
N, p=q
\end{array}\right.
$$

As VCF are N-periodic functions, then the matrix can be rewritten with a minimum phase generated after subtracting from $p x$ integer factor of $2 \pi$

The VCF system could be determined both at even and odd interval of $\mathrm{N}$.

VCF could be visualized at the plane as the rotating of unit length vector, whose projection on the abscissa and ordinate give the real and imaginary parts of the functions. The difference lies in the fact that in case of usual functions this vector rotates continuously, while in VCF it rotates abruptly.

\subsection{Matrix representation of VCF}

We will use only the aggregates of VCF being complete orthogonal systems, i.e. containing $N$ orthogonal functions defined in $N$ interval. Any such system of VCF could be represented as square orthogonal matrix of $N \times N$ size. CCF is a periodic function with $\mathrm{m}-$ based rational period, so they are determined on $N=m^{n}$ interval where $m$ and $n$ are integer numbers.

Alternation of functions in VCF system or arrangement of lines in VCF matrix may be different, which gives the opportunity to build different systems of VCF. The example of such systems is the Kronecker system of VCF. The matrix of this system, which we will denote as $\operatorname{VKF}(m, n)$ is $n^{\text {th }}$ Kronecker factor of discreet exponential function $\mathbf{D}_{\mathbf{m}}$
Let us consider building the system of base functions on VCF basis where the base of $m=4$. For easier understanding of basic functions system formation let $n=2$. The initial matrix of discrete exponential functions has the form:

$$
\mathbf{D}_{\mathbf{m}}=\left(\begin{array}{llll}
w_{0} & w_{0} & w_{0} & w_{0} \\
w_{0} & w_{1} & w_{2} & w_{3} \\
w_{0} & w_{2} & w_{4} & w_{6} \\
w_{0} & w_{3} & w_{6} & w_{9}
\end{array}\right),
$$

where $w=e^{j \frac{2 \pi}{m}}=e^{j \frac{2 \pi}{4}}$.

The constructive matrix is $\mathrm{n}^{\text {th }}$ Kronecker factor of discrete exponential matrix with $m \times m$ size.

$$
\operatorname{VKF}(m, n)=D_{\mathrm{m}}^{(\mathrm{n})},
$$

where $(n)$ means Kronecker factor of $\mathbf{D}$ matrix.

According to (7) the required basis for $m=4$ and $n=2$ will be represented as the matrix:

$$
\mathbf{V K F}(m, n)=\left(\begin{array}{cccc|c|cccc}
w_{0} & w_{0} & w_{0} & w_{0} & & w_{0} & w_{0} & w_{0} & w_{0} \\
w_{0} & w_{1} & w_{2} & w_{3} & & w_{0} & w_{1} & w_{2} & w_{3} \\
w_{0} & w_{2} & w_{4} & w_{6} & \cdots & w_{0} & w_{2} & w_{4} & w_{6} \\
w_{0} & w_{3} & w_{6} & w_{9} & & w_{0} & w_{3} & w_{6} & w_{9} \\
\hline w_{0} & w_{0} & w_{0} & w_{0} & & w_{0} & w_{0} & w_{0} & w_{0} \\
w_{0} & w_{1} & w_{2} & w_{3} & & w_{0} & w_{1} & w_{2} & w_{3} \\
w_{0} & w_{2} & w_{4} & w_{6} & \cdots & w_{0} & w_{2} & w_{4} & w_{6} \\
w_{0} & w_{3} & w_{6} & w_{9} & & w_{0} & w_{3} & w_{6} & w_{9}
\end{array}\right)
$$

The resulting matrix is symmetric, i.e. its properties are the same for variables $p$ and $x$. This property allows to use this transformation in forward and reverse directions without performing the transpose operation of matrices:

$$
C=\mathbf{V K F}(m, n) B \mathbf{V K F}(m, n)
$$

and

$$
B=\mathbf{V K F}(m, n) C \mathbf{V K F}(m, n),
$$

where $C$ - spectral coefficients matrix, $B$-image matrix.

\subsection{Using spectrum restriction in VCF base for image segmentation}

Spectrum in VCF basis differs from the familiar Fourier spectrum because the component corresponding to the highest frequency falls into the lower-right corner when using the ordering of the spectrum by frequency. VCF spectrum has no symmetry and all of its components have a small amount of information about the image.

Thus, in harmonic analysis in general case we have to face the necessity either to analyse complex components, or consider both the amplitude and phase spectrum to obtain a complete picture. The advantage of VCF spectrum is that most of the information about the image carries the amplitude spectrum.

Let's describe the impact of signal spectrum limitation. Spectrum of processed image can be defined by the formula

$$
C(u, v)=\hat{H}(u, v) \cdot B(u, v),
$$


where $\hat{H}(u, v)$ - two-dimensional discrete function of frequency attenuation; multiplication is performed elementwise.

Attenuation function of frequency in the simplest case for two dimensional ideal high pass filter has the form:

$$
\hat{H}_{f}(u, v)=\left\{\begin{array}{l}
1, \text { если } D_{f}(u, v) \geq D_{f}, \\
0, \text { если } D_{f}(u, v)<D_{f},
\end{array}\right.
$$

where $D_{f}$-edge frequency, $D_{f}(u, v)$-distance from point $(u, v)$ to the beginning of coordinate system.

In VCF spectrum various functions of attenuation corresponding to different variants of two-dimensional filter attenuation of lower frequencies can be offered. Figure 1 shows the diagram of three such variants.

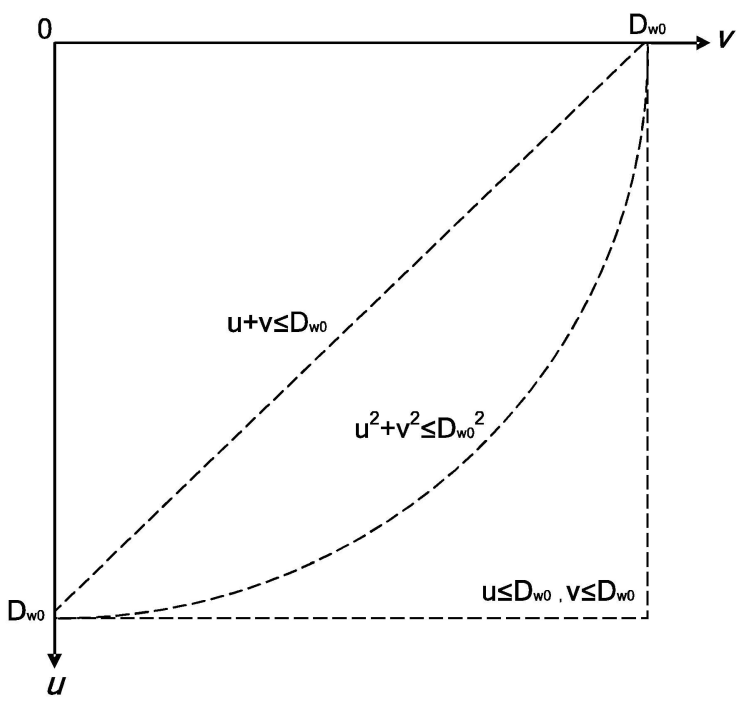

Figure 1. Two-dimensional area where three different borders of three filters are marked

Attenuation function for triangular region restriction option has the form:

$$
\hat{H}_{w}(u, v)=\left\{\begin{array}{l}
1, \text { если } u+v \geq D_{w}, \\
0, \text { если } u+v<D_{w} .
\end{array}\right.
$$

Attenuation function for round region restriction option has the form:

$$
\hat{H}_{w}(u, v)=\left\{\begin{array}{l}
1, \text { если } u^{2}+v^{2} \geq D_{w}^{2}, \\
0, \text { если } u^{2}+v^{2}<D_{w}^{2} .
\end{array}\right.
$$

Attenuation function for square region restriction option has the form:

$$
\hat{H}_{w}(u, v)=\left\{\begin{array}{l}
1, \text { если } u \leq D_{w} \text { и } v \leq D_{w}, \\
0, \text { если } u>D_{w} \text { или } v>D_{w} .
\end{array}\right.
$$

\section{Experiment results}

According to this example you can build a VCF system with any fiyld of definition $N$. Taking into account (2) the basis for $m=4$ and $n=4, N=256$ was built, its fragment is given in table 1. This fragment shows that if $m=4$ the VCF functions elements are complex.
For research the package of applied programs MATLAB was used, using it in research and image processing was described in [8-10].

Table 1. Formed basis matrix fragment

\begin{tabular}{|c|c|c|c|c|c|}
\hline $1+0 \mathrm{i}$ & $1+0 \mathrm{i}$ & $1+0 \mathrm{i}$ & $1+0 \mathrm{i}$ & $1+0 \mathrm{i}$ & $1+0 \mathrm{i}$ \\
\hline $1+0 \mathrm{i}$ & $0+1 \mathrm{i}$ & $-1+0 \mathrm{i}$ & $0+1 \mathrm{i}$ & $1+0 \mathrm{i}$ & $0-1 \mathrm{i}$ \\
\hline $1+0 \mathrm{i}$ & $-1+0 \mathrm{i}$ & $1+0 \mathrm{i}$ & $-1+0 \mathrm{i}$ & $1+0 \mathrm{i}$ & $-1+0 \mathrm{i}$ \\
\hline $1+0 \mathrm{i}$ & $0+1 \mathrm{i}$ & $-1+0 \mathrm{i}$ & $0-1 \mathrm{i}$ & $1+0 \mathrm{i}$ & $0+1 \mathrm{i}$ \\
\hline $1+0 \mathrm{i}$ & $1+0 \mathrm{i}$ & $1+0 \mathrm{i}$ & $1+0 \mathrm{i}$ & $0-1 \mathrm{i}$ & $0-1 \mathrm{i}$ \\
\hline $1+0 \mathrm{i}$ & $0-1 \mathrm{i}$ & $-1+0 \mathrm{i}$ & $0+1 \mathrm{i}$ & $0-1 \mathrm{i}$ & $-1+0 \mathrm{i}$ \\
\hline
\end{tabular}

Test image fragment and its spectrum in VCF basis are presented at Figure 2.

Different types of spectrum fields limits (square, round, triangular) application, according to obtained experimental data did not lead to a significant difference between the results of their application. For further research of spectrum restriction effects was selected round area, as more comparable to the traditional method based on the Fourier basis.

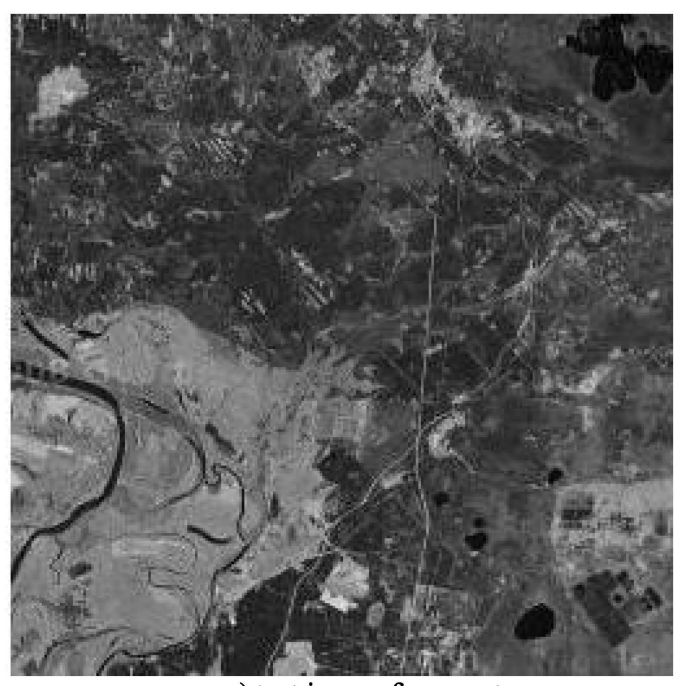

a) test image fragment

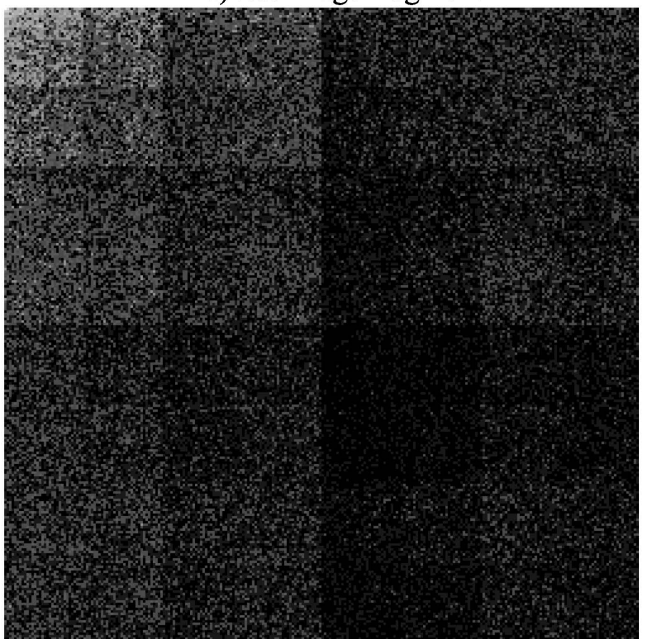

b) VCF spectrum

Figure 2. Test image fragment and its VCF spectrum in constructed basis.

During experimental studies, we analyzed the possible options of spectrum limit. Only high- or only low- 
frequency spectrum components restrictions did not give the desired results, however, the combination of simultaneous constraints high-frequency and lowfrequency components gave results that together with the correction of the resulting image gave a result that can be used to highlight borders of informative regions in image sensors vision systems.

The results of the application so-called band-pass filters that limit the spectrum simultaneously with the upper and lower frequencies are shown at figure 3.

Restored image highlights areas characterized by comparable values of brightness at the respective samples. The line features are aggravated. This property can be used to select informative elements during the navigation systems data preliminary preparation.

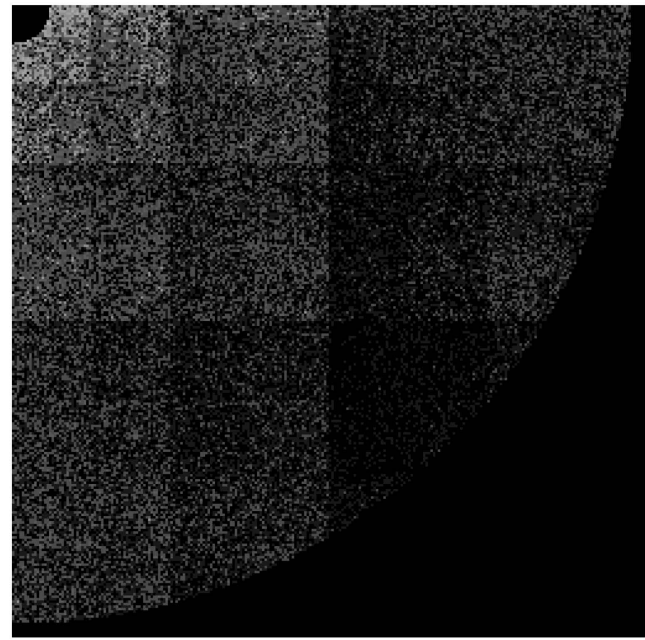

a) restricted spectrum

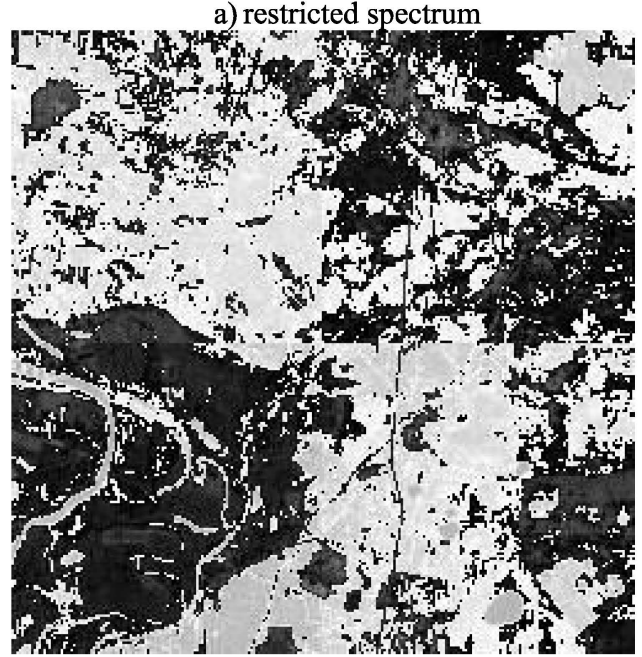

b) restore image fragment

Figure 3. Band-pass filtering results

Limit frequencies boundaries of described bandpass filter may vary based on the need to highlight or detract from certain image properties.

Changing boundary frequencies areas research showed the ability of band-pass filters to clearly identify comparable areas of small extent. The sensitivity of filter to such objects is determined by the frequency of the lower cutoff filter. The average brightness within areas characterized by an upper frequency cutoff of the filter.
The results of the changing cutoff frequencies are shown at Figure 4.

Restricted spectrum filters ability to highlight small areas can be applied in image processing where pixel brightness characterizes the density of the investigated object that can be applied in many related areas: radiography, medicine and so on.

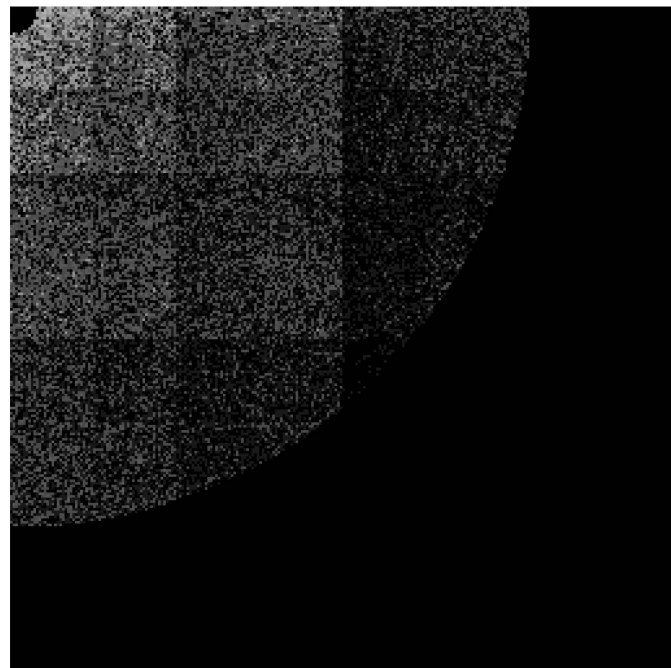

a) restricted spectrum

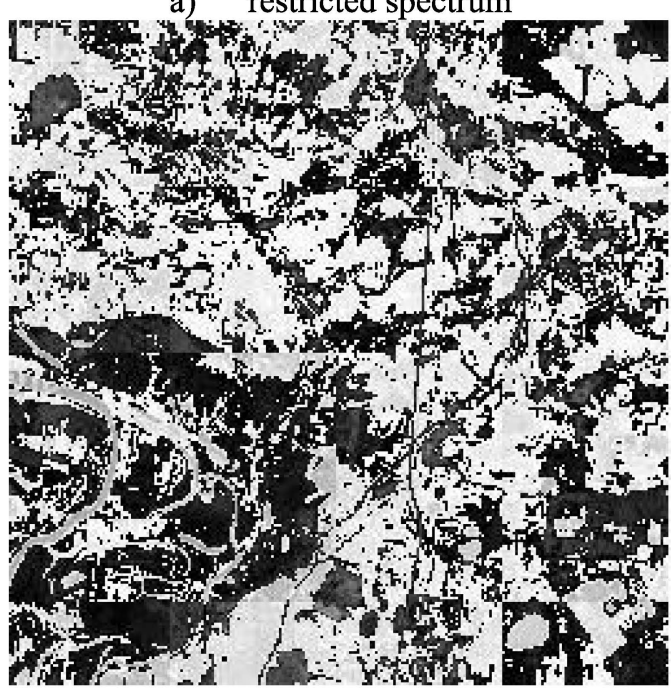

b) Borestore image

Figure 4. Band-pass filtering results

\section{Conclusion}

To compare the results of applying restrictive filters we used already studied Fourier basis and execute in it similar transformation of the spectra. The obtained restored image are comparable to the compliance using one of images difference measurement metrics. Similar images will be considered images, if difference between them is less than some threshold. Currently, the most popular and common metrics used to evaluate quality of restored images is the root mean square error (RMSE). MSE can be calculated by the formula:

$$
\sigma=\sqrt{\frac{1}{M N}} \sum_{x=0}^{M-1 N-1} \sum_{y=0}(\hat{f}(x, y)-f(x, y))^{2} .
$$


Comparative characteristics for VCF basis to the Fourier basis are shown in table 2. The purpose of this experiment is as follows: in the limitation spectrum of the image four times around the same time the resolution must deteriorate. When you drop limited of spectral components, the image size is reduced four times. In the same spatial area of the image as elements of this image will be the pixel with the average brightness that can be taken as the standard of correctness of the algorithm.

Table 2. Comparative characteristics

\begin{tabular}{|c|c|c|}
\hline Base & VCF & Fourie \\
\hline Quantity of differentiae pixels & 123 & 117 \\
\hline Mean squre root error (MSE) & 0,1589 & 0,1154 \\
\hline Executing time, s & 0,0151 & 0,0586 \\
\hline
\end{tabular}

Analysis of data in table 2 shows that the proposed method can perform the same functions as the Fourier basis, with comparable quality of results.

\section{References}

1. A.N. Kolesenkov, B.V. Kostrov and V.N. Ruchkin, 2nd Mediterranean Conference on Embedded Computing, MECO 2013, Emergencies monitoring and preventing Proceedings, pp. 263-265 (2013)

2. A. Kolesenkov, B. Kostrov, E. Ruchkina and V. Ruchkin, 3rd Mediterranean Conference on Embedded Computing, MECO 2014, Anthropogenic situation express monitoring on the base of the fuzzy neural networks Proceedings, pp. 166-168 (2014)

3. A.N. Kolesenkov, B.V. Kostrov, V.N. Ruchkin and E.V. Ruchkina, 4th Mediterranean Conference on Embedded Computing, Algorithms of Fire seat Detection, Modeling Their Dynamics and Observation of Forest Fires via Communication Technologies, pp.254 -257 (2015)

4. S.E. Umbaugh, Computer Imaging: Digital Image Analysis and Processing (CRC Press, 2005)

5. M. Seul, L. O'Gorman and M. J. Sammon, Practical Algorithms for Image Analysis (Cambridge, 2000)

6. M. Petrou, P. Bosdogianni, Image Processing: The Fundamentals (John Wiley \& Sons, 1999)

7. S.W. Smith, The Scientist and Engineer's Guide to Digital Signal Processing (California Technical Publishing, 1999)

8. W.L. Martinez, A.R. Martinez, Exploratory Data Analysis with MATLAB (CRC Press, 2005)

9. F. Gustafsonand, N. Bergman, MATLAB for Engineers Explained (Springer, 2003)

10. C.F. Van Loan, Introduction to Scientific Computing: A Matrix-Vector Approach Using $M A T L A B$ (Prentice Hall, 1999) 